\title{
PERIPHERAL ARTERIAL DISEASE
}

\section{Some Points of Common Interest to General and Orthopaedic Surgery}

\author{
F. A. R. Stammers, Birmingham, England \\ Professor of Surgery. Cniversity of Birmingham
}

Before 1926 the popular method of combating vasospasm was by periarterial sympathectomy, as advocated by Lériche; but the benefit was only temporary, and therefore disappointing. The first lumbar sympathetic ganglionectomy for peripheral arterial disease in the lower limb was performed twenty-seven years ago by Adson and Brown (1929) at the Mayo Clinic, and the genesis of the operation is of interest. During the early twenties of this century Hunter (1924) in Australia was conducting experiments in support of his hypothesis that striped muscle had a dual innervation-the position of a limb being altered through the medium of somatic nerves, and the new position being maintained through sympathetic nerves-and that spasticity of the legs from injury to the spinal cord at a higher level was evidence of overaction of the sympathetic. Acting on this hypothesis, Royle (1924), a surgical colleague, performed lumbar sympathetic ganglionectomy on soldiers suffering from spastic legs, the result of wounds in the first world war. Royle claimed good results, and he was invited to America to demonstrate his operation. Among other centres, he visited the Mayo Clinic where he was offered a group of these cases. The spasticity was little affected, but what was of particular interest to the observers was the fact that the legs became warmer and drier than hitherto. It was at that time that Allen and Brown were engaged on a study of peripheral vascular disease, and they realised at once that here was something that offered great possibilities where vasospasm played a part in the symptoms. Adson and Craig of the neurosurgical department adopted the operation for just these cases. Thus the discovery of the effect of sympathectomy was accidental: it was an example of " the mind prepared."

There was still no satisfactory surgical approach to the stellate ganglion until the rediscovery of Henry's paper of $\mathbf{1 9 2 3}$ on a method of ligating the first part of the left subclavian artery from behind, in which he mentioned that the ganglion could be seen through a dorsal incision through the bed of the second rib. The first time that this was undertaken for peripheral arterial disease of the upper limb was again by Adson, during the latter part of 1928, and from this time onwards sympathetic ganglionectomy became one of the most useful procedures for severe vasospasm (Stammers 1932, 1944). It was my good fortune to spend the whole of 1929 at the Mayo Clinic working in the Department of Neurosurgery.

Classification of peripheral arterial diseases-Peripheral arterial diseases can be listed in five main groups: 1) Spasm alone (mainly in young people): poliomyelitis; acrocyanosis frigida; Raynaud's disease; and an unclassified group of vasospasm. 2) Anatomical (usually, but not always, in young people): cervical rib, and mechanisms within the costo-clavicular space. 3) Arterial wall disease with or without superimposed spasm; Buerger's disease (young patients); endarteritis (diabetic; syphilitic); medial sclerosis (middle-aged patients); atherosclerosis (old patients); aneurysm. 4) Embolism. 5) Trauma: blunt injuries such as mechanical blow or crutch pressure; open wound-especially gunshot wounds; thermal: frost bite; immersion foot.

\section{SYMPTOMS OF VASGULAR DISEASE}

The symptoms that should arouse suspicion of underlying peripheral arterial disease may be considered under the following headings. 
Cold sensitivity-Some young men and women always have cold hands and feet, often looking cyanosed too. They are perfectly healthy, and do not suffer any discomfort. They are just more sensitive to cold than most people, but if in them, or as a sudden or gradual thing in older folk, this coldness becomes painful, real vascular disease must be assumed.

Trophic changes-These take various forms: nails may become deformed; cracks may appear near the nail margins; ulcers may develop on the tips of fingers or toes; or frank gangrene may involve one or more digits. Sometimes a surgical or accidental wound of fingers or toes fails to heal and peripheral vascular disease is then suspected for the first time. For example, there are now two patients in my wards, one because the operation wound for ingrowing toe nail failed to heal, and the second because a foot injured by a falling weight showed no evidence of healing.

Intermittent claudication - This intermittent limp, the result of severe cramp from ischaemia of muscles, may affect calf, peroneal, thigh or foot muscles, the first being the commonest in my experience. Claudication affecting the thigh muscles is caused by blocking of the profunda femoris artery: when the small muscles of the foot are affected the pain may at first mimic foot strain, and foot exercises or foot supports may be mistakenly given.

A mixture of symptoms is complained of by some patients - claudication, trophic changes, and coldness all appearing at the same time and often proceeding to gangrene.

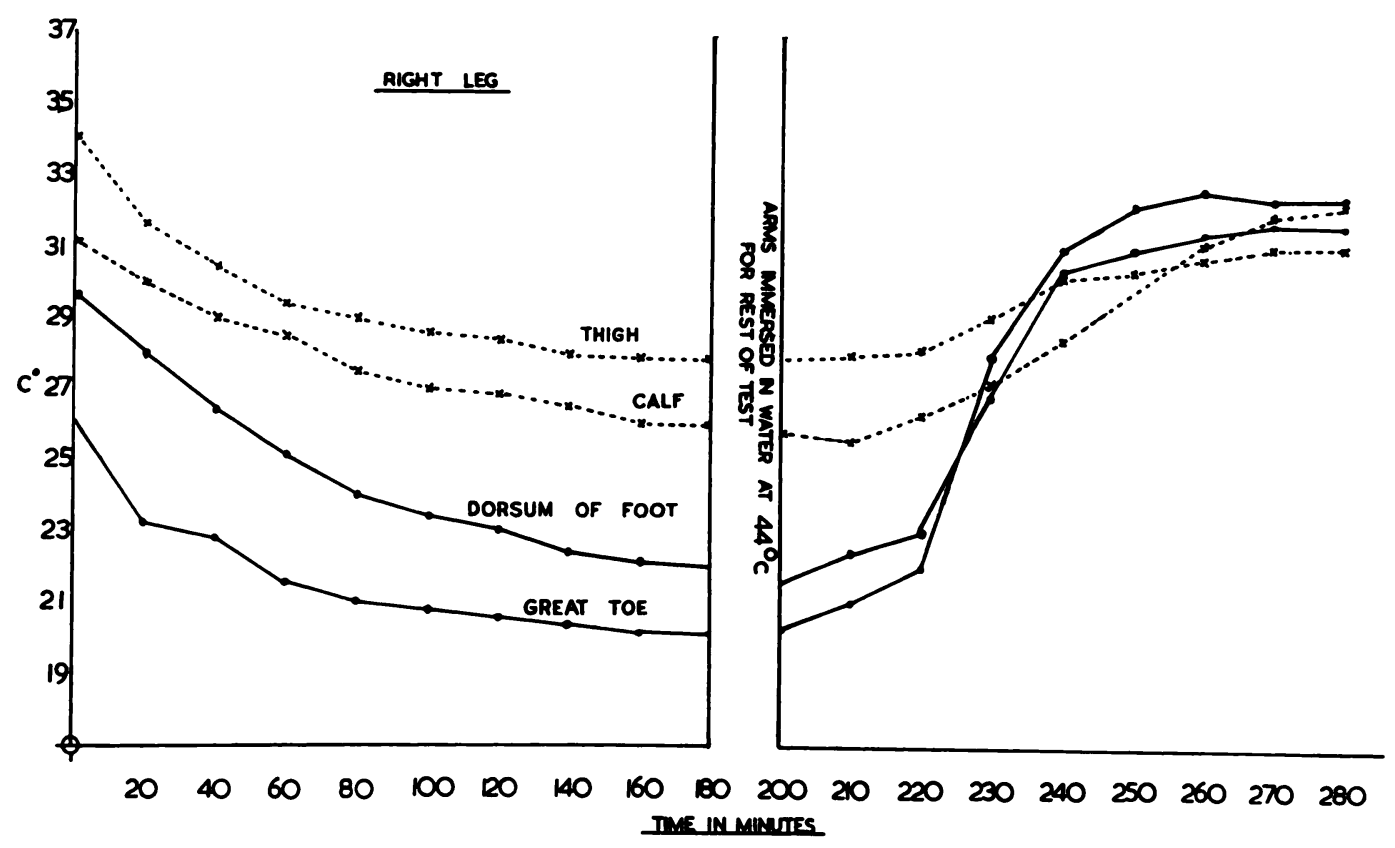

FIG. 1

Heating test (right leg). On the left are temperatures whilst the limbs lay exposed to environmental temperature: on the right are the results of heating the arms. The findings for the left leg were similar. After twenty minutes the body temperature had risen from its resting level of 36.8 degrees Centigrade to 37.5 degrees Centigrade. (Room temperature 19.2 degrees Centigrade.)

\section{INVESTIGATIONS}

When disease of the peripheral vessels is suspected investigation is required, first to prove the presence of disease, and secondly - and most importantly - to assess the degree of vasospasm, for it is this and this alone that can be influenced by operations on the sympathetic nervous system.

History and clinical examination-The history of onset, the age and general condition of the patient, and an examination of the peripheral pulses, all help in diagnosis. But it must be 
remembered that many a patient's peripheral arteries contract down under the influence of a cold journey so that pulses are eliminated; such patients should be allowed to warm up for a few hours before a firm statement is made that there are no pulses distal, for instance, to the groin.

In my experience one of the most useful questions to the patient is: " do your feet warm up in bed at night?" If they do so spontaneously it is certain that vasospasm plays a part in the picture.

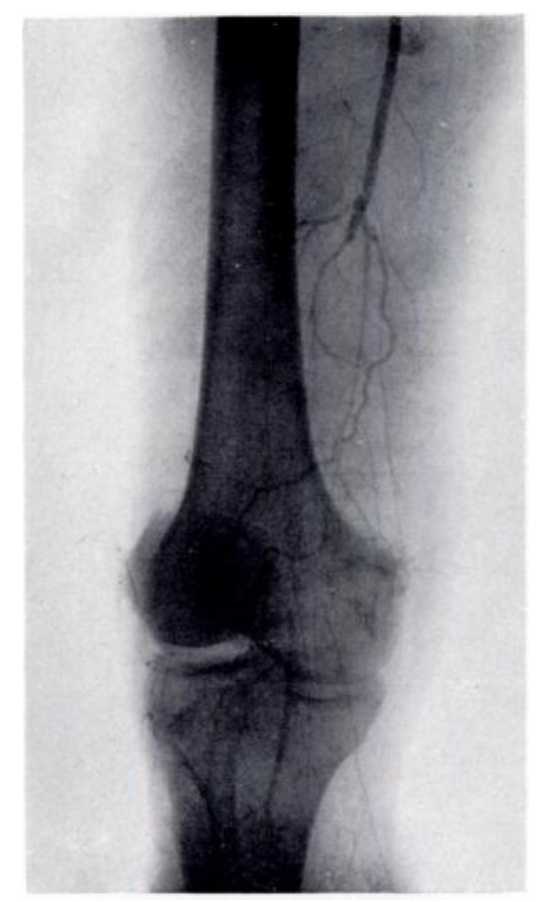

FIG. 2

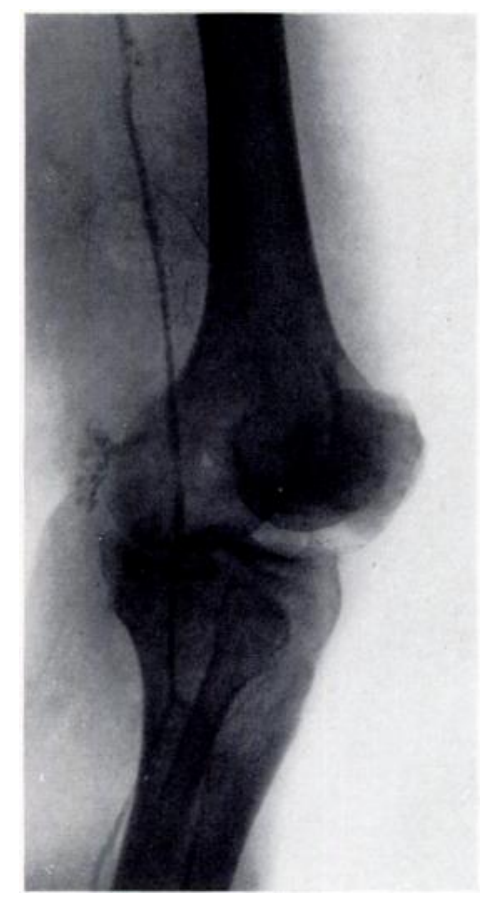

FIG. 3

Figure 2-Arteriogram showing block in popliteal artery. Figure 3-same case after vein graft.

I:ffect of reflex heating-The physiological stimulus to a general vasodilation of the limbs is prrexia, and a mild, harmless pyrexia can be produced in several ways. If the legs are affected, the hands and forearms up to the elbows can be immersed in water at $\mathbf{4 4}$ degrees Centigrade (as hot as can be borne), which raises the mouth temperature after half an hour or an hour to 36.5 or 37 degrees Centigrade. If the hands are the affected parts, the legs up to just below the knees are immersed in the hot water. The full test consists in exposing the affected limbs to room temperature (preferably at 15.5 degrees Centigrade) for about one hour, at the same time taking mouth temperature, and then heating the normal limbs, taking temperatures of a digit, the dorsum of foot or hand, and of the calf or forearm, again observing mouth temperature as an indication of a mild pyrexia. The peripheral temperatures are most conveniently measured by electro-thermal couples, but an ordinary short thermometer, graduated from 20 to 40 degrees Centigrade, will serve. In a case of poliomyelitis or of Raynaud's phenomenon a rise of as much as 10 or 12 degrees Centigrade follows reflex heating, whereas an elderly patient with arterio-sclerosis may show no more than a rise of 0.5 to 2 or 3 degrees Centigrade: these changes are commensurate with the degree of spasm (Fig. 1). A rougher test is to heat the trunk under an electric cradle; but it is not very accurate.

Sympathetic block-The best mimicry of a surgical sympathectomy is to inject a solution of $\because$ per cent procaine around the appropriate ganglia. The procedure is technically simple, 
and its effectiveness is determined by similar temperature studies. I do not advocate the use of spinal anaesthesia as a test, although it is the most effective method of producing temporary paralysis of the sympathetic outflow from the cord: it is not free from risk, and such catastrophes as loss of sphincter control or

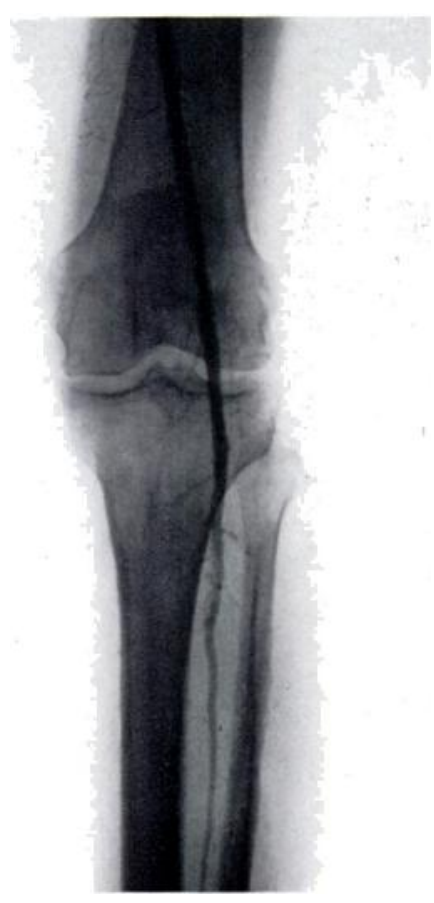

FIG. 4

. Irteriogram showing good though irregular lumen in arterial tree. Despite this there was gangrene of toes and a below-knee amputation stump failed
to heal.

drop-foot have been observed.

Arteriography - This has assumed great importance since the introduction of arterial grafting techniques. Nevertheless, its interpretation is sometimes difficult, and Morrissey, working in my department, has shown that normal people may reveal a kink, or even nonfilling of a segment at points where the vessels pass through anatomically narrower or tethered regions, such as the adductor opening or the popliteal space. Figures 2 and 3 show the typical appearance of a blocked femoral artery replaced by a vein graft (by D. M. Morrissey). Figure 4 shows femoral and popliteal arteries which appear to have a good though somewhat irregular lumen; the patient was a man of seventy-five with arterio-sclerosis and gangrene of toes. Because of the arteriogram a below-knee amputation was ventured, but the stump failed to heal, and even the subsequent above-knee stump healed only sluggishly. I have tried arteriograms in a number of patients requiring amputation, in the hope that they would help in assessing the chance of a successful below-knee amputation, but I have not found them a reliable guide. I believe that the only reliable method in the presence of peripheral arterial disease is to make the incisions boldly as for the below-knee amputation and to proceed according to the profusion of the bleeding.

Other investigations-What is really needed when claudication is the presenting symptom is some electro-mechanical device which, when plunged into different muscle bellies under local anaesthesia, will measure change in blood-flow before and after sympathetic paralysis. Morrissey has, like others, tried measuring the uptake of radio-active sodium injected into the calf muscle before and after sympathetic block, but the results are equivocal.

\section{TREATMENT}

The object of treatment is to eliminate such arteriospasm as there is, and so open up the collateral circulation. Treatment may be conservative or operative.

Conservative treatment-Smoking of cigarettes should be absolutely forbidden. Alcohol, twice daily, is not only valuable but usually greatly welcomed-stout for the ordinary patient, spirits within moderation for the well-to-do.

Drugs-Anti-spasmodic drugs such as " Priscol " and nicotinic acid, ganglion-blocking agents such as the methonium group of drugs, and "Padutin" all help. The last is a non-insulin extract of pancreas possessing vasodilator properties. It is not standardised and, therefore, not acceptable to some authorities, but I know from experience that it is often most helpful. A combination of two of these drugs is worth while.

Exercises-Buerger's exercises are beneficial and make the patient feel that he is doing something towards his own treatment. 
Reflex heating-Reflex heating is most useful, and can be carried out in the home by the patient immersing the hands and forearms in as hot water as he can tolerate, for ten minutes twice daily. A method that I have used now for over five years for in-patients is to make them wear electrically heated gloves extending up to the elbows for many hours each day. Indeed they need be removed only for meals. Recently we have used a battery of such gloves as shown in Figure :).

Adjustments to footriear-Raising the heels of the shoes relieves the demand on the calf muscles and gives relief.

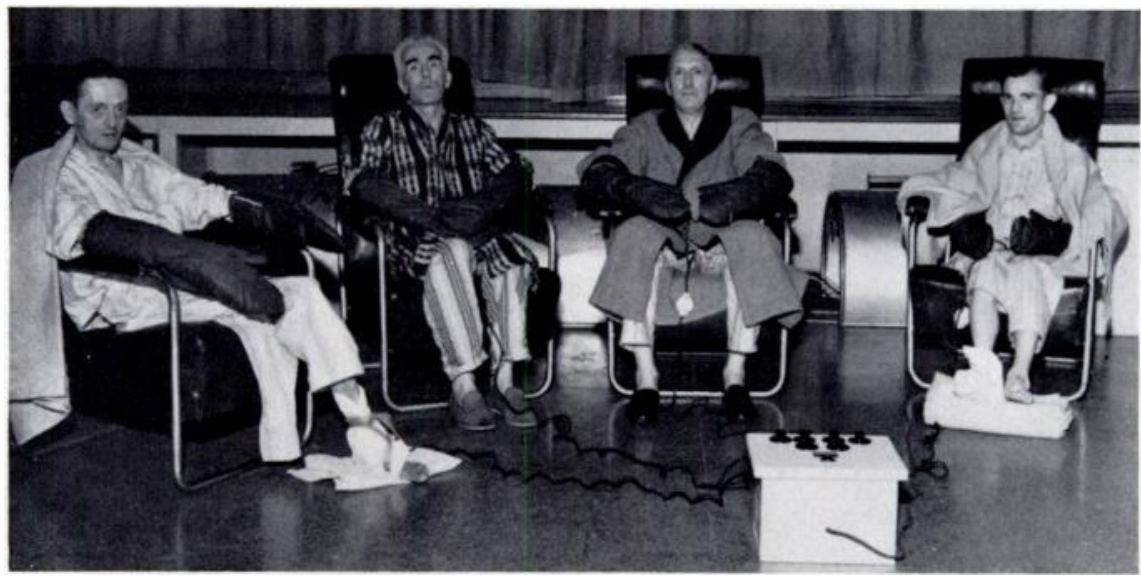

FIG. 5

Reflex heating by electrically heated gloves.

Operative treatment. Sympathectomy-The techniques of sympathectomy are well known and I will merely make a few comments. The operation for the lower limbs-removal of lumbar ganglia 2,3 and 4 with intervening chain - if required on both sides, can be carried out at the same operation through bilateral oblique lateral abdominal incisions down to the peritoneum. This can then be stripped medially with little fear of subsequent paralysis of bowel, a risk one might think likely with such extensive stripping on both sides. Meteorism should be watched for, and if threatened can be treated by gastric intubation and parenteral feeding for a few days. I have seen it only twice, and it responded quickly to treatment.

The operation for the upper limb is less certainly effective than that for the legs, because the anatomy is much more variable (Mitchell 1953). For this reason there are several different approaches; all are straightforward. The Smithwick (1940) approach through the bed of the third rib behind can be followed by an extra-pleural or intra-pleural operation, the second and third thoracic ganglia with intervening chain being excised. Some surgeons have also removed the first, this being the lower half of the stellate ganglion. Horner's syndrome is not produced if the upper half of the stellate ganglion - that is, its inferior cervical moietybe preserved. Should relapse follow the more orthodox operation, Poppen (1950) advocated extension of the excision down to the fifth ganglion. The orthodox ablation can also be carried out from above the clavicle and through Sibson's fascia, as described by Telford (1938), and this is useful when it is not certain until exploration whether vasospasm of an upper limb is due to a cervical fibrous band or is idiopathic; should there be no band, sympathectomy may be proceeded with through the same operation field. A third technique, initiated in South Africa, is through the bed of the third rib in the axilla and then across the apex of the thoracic cavity.

Tenotomy of the tendo calcaneus - I have little experience of this operation introduced by Boyd et al. (1949), but I have carried it out in three old men, converting a painful hobble into a painless shuffle. They felt it worth while.

vol. $36 \mathrm{~B}$, No. 2 , MAY 1954 
Arterial grafting-This is in its trial period, but the recent reports of Brock (1953) and Rob (1953) made thrilling reading. That some grafts thrombose subsequently there can be no doubt, but the graft can be repeated, and even a year's respite from pain and threatened loss of limb is worth while. Arterial banks are being established in most centres, and the freeze-dry method of preservation makes transport of grafts an easy matter. Veins may be used, too, and in traumatic cases may be better because available and autogenous, but care must be taken to put them in upside down, otherwise the valves would nullify the intention.

Reports from Korea vary from high hope to great caution. Apart from thrombosis there is an anxiety that these arterial homografts may at a later date blow out as aneurysmal dilations. Much has to be learned yet of this hopeful form of treatment.

\section{COMMENTS ON INDIVIDUAL DISEASES}

Poliomyelitis-Some patients suffer, within a year or two of the acute phase, severe vasospasm of the affected limbs. They are cold, blue or mottled, and these symptoms are roughly commensurate with the degree of paralysis. The patients that I see are sent by orthopaedic colleagues usually two or three years after the initial disease because the victims begin to get real discomfort during the winter months; the coldness amounts to frank pain, and usually trophic ulceration develops around the ankle or on the dorsum of the foot. Never does a digit become gangrenous. Often this ulceration renders these patients bedridden during winter. Yet in a warm room or bed they warm up spontaneously. Reflex heating tests show a marked rise of temperature, even as much as 15 to 20 degrees Centigrade. Quite often I am asked to operate with the express purpose of improving the blood supply for a subsequent arthrodesis. The cold-sensitivity is caused by excessive vasospasm from the lack of the vascular pump effect of active muscles. Sympathectomy leads to a warm, dry and pink limb, and the trophic ulcers heal, at least for a time: but this is not the end of this happy story. When arthrodesis is subsequently performed for a flail foot, the forefoot often becomes cold again though remaining dry, probably because of further deprivation of the venous pump effect; and when the knee is arthrodesed too, the leg becomes still more cold-sensitive from mid-calf downwards. Probably the excessive accumulation of fat, with its poor blood supply, is also important, and trophic ulceration always overlies the fat.

Acrocyanosis frigida-This malady affects young females with fat ankles, and it is this cuff of fat surrounding the ankles that is cold-sensitive and becomes blue and mottled as in poliomyelitis. In a warm room or in bed they become comfortable and of normal colour. This stage proceeds to trophic ulceration in the fatty zone, affecting front and back of the ankle; the toes are unaffected. The disease is again an example of ischaemia of fat. Ulceration may be preceded by subcutaneous tender nodules; biopsy reveals giant-cell systems which are foreign body reactions to dead fat, and not tuberculous.

Bazin's disease-This must be mentioned because it, too, presents small chronic ulcers, but only over the tendo calcaneus, and without excessive fat. This is truly a tuberculous infection. The ulceration of Bazin's disease is not improved by sympathectomy.

Cervical rib-The possible symptoms from a cervical rib are usually divided into motor, sensory and vascular. I have had well over sixty cases of cervical rib or costo-clavicular space syndrome, and have pointed out (1950) that vascular symptoms occur only in the presence of a highly arched subclavian artery; this lies in contact with the extra rib and is pressed against it by each contraction of the scalenus anterior muscle. Since this is an accessory respiratory muscle it contracts, in the average person, about 20,000 times each day. Vascular injuries-This subject gives so many varieties of vascular lesions as to require a book; a few examples only will be offered.

Blunt injuries-A sudden blow with a blunt object may bruise an artery, throwing it into spasm and thereby embarrassing the blood supply to the limb. Four years ago Mr W. Gissane invited me to see a young man who, while conveying on a motor lorry metal ingots from one 
end of a large shed to the other, backed into an $\mathrm{H}$-girder, the edge of which caught him behind the knee. He sustained mild bruises in the popliteal space, but the foot and lower leg rapidly became cold, white and insensitive, and he was taken to the Birmingham Accident Hospital. There, every stimulus to peripheral vasodilation was applied, and in about six hours the circulation returned. Had it not done so, it would have been necessary to explore the popliteal artery; and to-day, thanks to the researches of Kinmonth (1952), 2 per cent papaverine

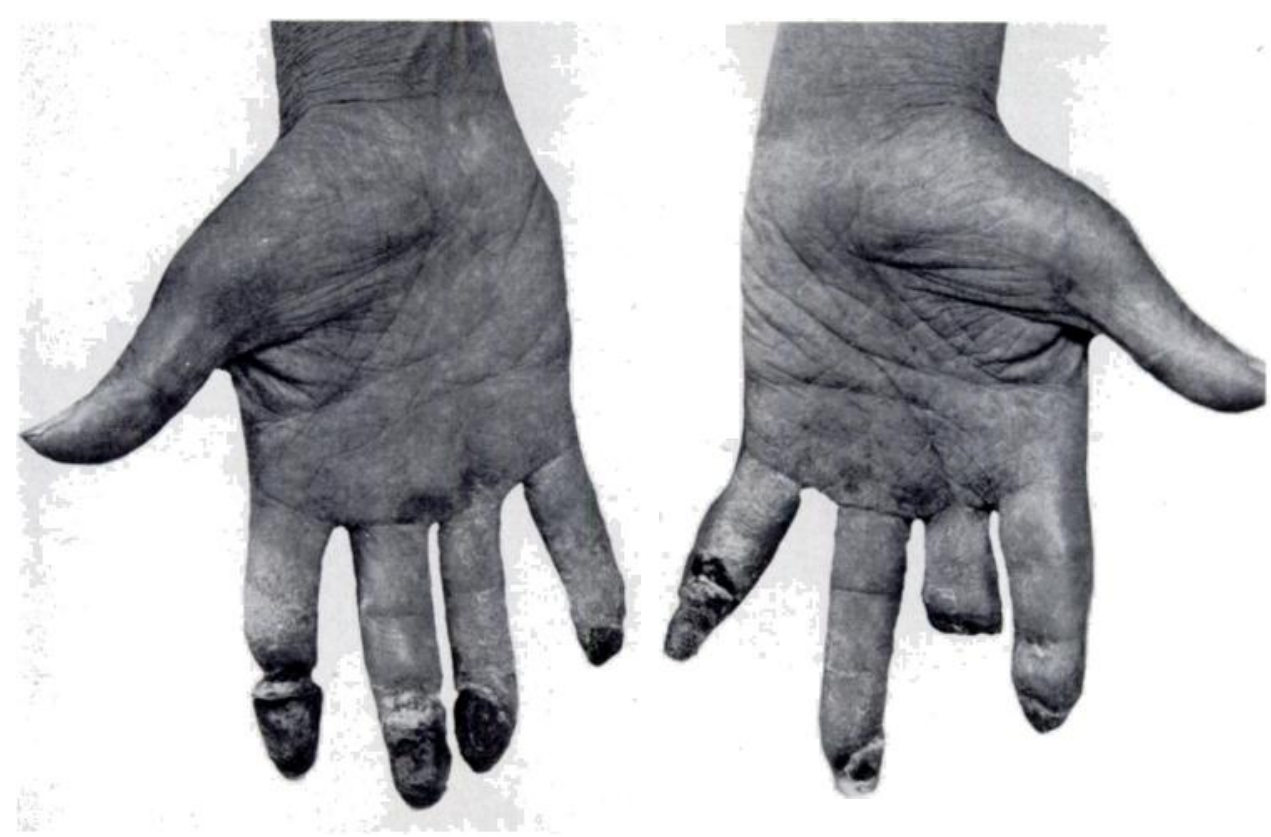

Fig. 6

Arteritis from crutch pressure causing gangrene of several digits.

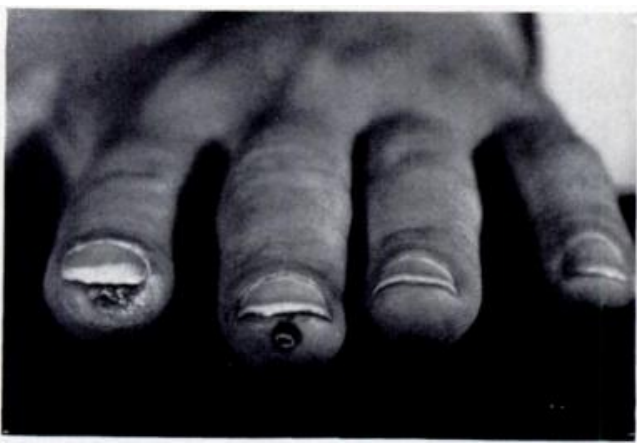

FIG. 7

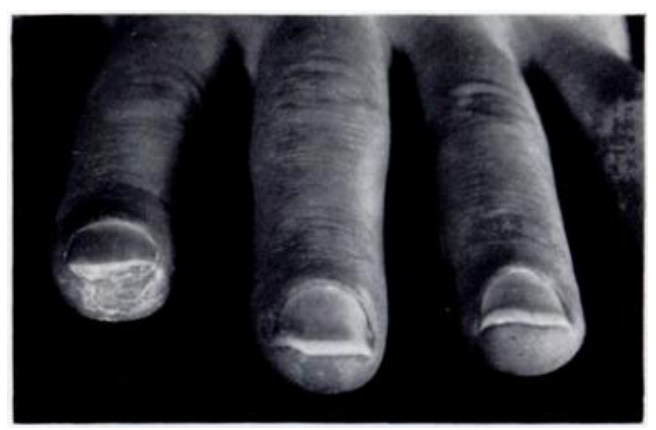

FIG. 8

Figure 7- Case of crutch pressure arteritis causing finger-tip gangrene of left hand. Figure 8 -Same case twenty days after cervico-thoracic sympathectomy.

would have been painted on the artery or instilled around it continuously through a polythene tube. Papaverine has often been found to be effective in traumatic spasm when all other drugs have failed.

Other injuries-Severance of the popliteal or femoral artery is a serious matter. During the recent war I collected notes of such cases: twenty-six of thirty-six patients with injuries of the popliteal artery required amputation above the knce, and of thirty-one patients with femoral artery injuries twenty-one needed amputation. These are the cases in which arterial or venous grafts would now be used.

VOL. 36 B, NO. 2, MAY 1954 
Crutch arteritis-Four patients with crutch arteritis have been seen in the last six years. All had lost a lower limb during early childhood, and had never taken to a prosthesis. In each case, comparatively suddenly, serious circulatory embarrassment developed in one or both hands, which became cold and white, with loss of sensation and of function. All were treated on the same principle, namely, "turning on all the taps." At first the effect was tried of reflex heating, "Priscol" and alcohol, peristellate injection of 2 per cent procaine. These measures were followed next day by sympathectomy. Finally, after a true assessment of what recovery could be expected, such fingers as were gangrenous were amputated (Fig. 6). It is surprising how much recovery will occur. The photographs show the hands of two such cases (Fig. 7 and 8).

Embolism-Students should be taught that unless embolectomy is performed within three to four hours the limb will inevitably be lost. So often the physician or family doctor thinks that because the patient has heart disease he would not stand the journey to hospital, and certainly not a subsequent anaesthetic; these fears are of course unfounded.

\section{RESULTS OF SYMPATHEGTOMY}

The fundamental question to answer is how long after sympathectomy a limb will remain warm and dry. Of twelve patients whom I have observed for fifteen to twenty-two years, seven still have warm and dry limbs. The greater the element of spasm, the more successful the result. But many patients have disease of the vessel walls; this progresses-in Buerger's disease relentlessly so until all four limbs may be involved, though mercifully there is a tendency for this disease to be self-limiting. The comfort of poliomyelitis patients varies inversely with the deposition of fat, and may be interfered with by subsequent arthrodeses. The same applies to patients with acrocyanosis frigida: fat is always cold-sensitive, even after sympathectomy. Sympathectomy is always more certain in the lower limb than in the upper because of the more compact and constant anatomy; certainly $\mathbf{4 0}$ to 50 per cent of patients relapse after sympathectomy for the upper limb.

Nearly all those who complain of intermittent claudication at the age of fifty-five to sixty years, however treated, are dead of coronary disease within five years.

\section{SUMMARY}

1. The history of the development of the operation of sympathetic ganglionectomy for vasospasm is related.

2. A simple classification is given of the common diseases of the peripheral arteries.

3. The symptoms of peripheral arterial disease are described.

4. The investigations are discussed.

5. The treatment, both conservative and surgical, is discussed, with comments on arterial grafts.

6. Special points are made regarding poliomyelitis, acrocyanosis, Bazin's disease, cervical rib, vascular injuries and crutch arteritis.

7. The long-term results of sympathectomy are reviewed.

\section{REFERENCES}

Adsox, A. W. (1949): Cervical Ribs and the Scalenus Anticus Syndrome. In A Textbook of Surgery. Edited by F. Christopher. Fifth edition. Philadelphia and London: W. B. Saunders Company.

Adson, A. W., and Brown, G. E. (1925): Treatment of Raynaud's Disease. Journal of the American Medical Association, 84, 1,908.

Adson, A. W., and Brown, G. E. (1929): The Treatment of Raynaud's Disease by Resection of the Upper Thoracic and Lumbar Sympathetic Ganglia and Trunks. Surgery, Gynecology and Obstetrics, 48, 577. 
Boyd, A. M., Ratcliffe, A. Hall, Jepson, R. P., and James, G. W. H. (1949) : Intermittent Claudication. Journal of Bone and Joint Surgery, 31-B, 325.

BRock, R. C. (1953): Discussion on Reconstructive Arterial Surgery. Proceedings of the Royal Society of Medicine (Section of Surgery), 46, 115.

Henry, A. K. (1923): A Method of Ligaturing the First Stage of the Left Subclavian Artery from Behind. British Journal of Surgery, 10, 367.

Hunter, J. I. (1924): The Influence of the Sympathetic Nervous System in the Genesis of the Rigidity of Striated Muscle in Spastic Paralysis. Surgery, Gynecology and Obstetrics, 39, 721.

Kinmonth, J. B. (1952): The Physiology and Relief of Traumatic Arterial Spasm. British Medical Journal, i, 59.

Mitchell, G. A. G. (1953): Anatomy of the Autonomic Nervous System. Edinburgh and London: E. \& S. Livingstone Ltd.

Poppen, J. L. (1950): Personal communication.

Rов, C. G. (1953): Discussion on Reconstructive Arterial Surgery. Proceedings of the Royal Society of Medicine (Section of Surgery), 46, 121.

Royle, N. D. (1924): A New Operative Procedure in the Treatment of Spastic Paralysis and its Experimental Basis. Medical Journal of Australia, 1, 77.

Smithwick, R. H. (1940): The Rationale and Technic of Sympathectomy for the Relief of Vascular Spasm of the Extremities. New England Journal of Medicine, 222, 699.

Stammers, F. A. R. (1932): Some Experiences with Sympathetic Ganglionectomy. British Journal of Surgery, 20, 67.

Stammers, F. A. R. (1944): Peripheral Vascular Diseases, with Special Reference to those seen in the Fighting Forces. Medical Press and Circular, 211, 308.

Stammers, F. A. R. (1950): Pain in the Upper Limb from Mechanisms in the Costoclavicular Space. Lancet, i, 603.

TElford, F. D. (1938): Sympathetic Denervation of the Upper Extremity. Lancet, i, 70. 